\title{
Commentary: The mid-life crisis of the meta-analysis
}

\author{
Joanna Chikwe, MD, FRCS, ${ }^{\mathrm{a}, \mathrm{b}}$ and Aaron Weiss, $\mathrm{MD}^{\mathrm{a}}$
}

From the ${ }^{\mathrm{a} D e p a r t m e n t}$ of Cardiovascular Surgery, Mount Sinai Medical Center, New York; and ${ }^{\mathrm{b}}$ Department of Surgery, The State University of New York, Stony Brook, NY.

Disclosures: Jo Chikwe has received speaker honoraria for Edwards Lifesciences. Dr Weiss has nothing to disclose with regard to commercial support.

Received for publication Nov 13, 2018; accepted for publication Nov 14, 2018; available ahead of print July 4, 2019.

Address for reprints: Joanna Chikwe, MD, FRCS, Department of Cardiovascular Surgery, Mount Sinai Medical Center, 1190 Fifth Ave, New York, NY 10029 (E-mail: Joanna.Chikwe@mountsinai.org).

J Thorac Cardiovasc Surg 2019;158:716-7

$0022-5223 / \$ 36.00$

Copyright $(2018$ Published by Elsevier Inc. on behalf of The American Association for Thoracic Surgery

https://doi.org/10.1016/j.jtcvs.2018.11.045

The meta-analysis is 40 years old and having a mid-life crisis. ${ }^{1}$ Conceived in the 1970 s, the meta-analysis synthesizes studies to provide "best evidence" through systematic review, rigorous statistics, and formal protocols promoting reproducibility and reducing bias. However, success has not come without problems. A recent editorial in Nature observes: "One undesirable consequence of the growing recognition and high impact of meta-analysis is an increase in less-than-rigorous applications of these methods...Meta-analyses and systematic reviews can highlight areas in which evidence is deficient, but they cannot overcome these deficiencies-they are systematic and scientific techniques, not magical ones."

Which brings us to the meta-analysis in this issue of the Journal, in which Diaz and colleagues ${ }^{2}$ compare bioprosthetic with mechanical aortic valves in patients aged 50 to 70 years. The authors include 1 randomized and 4 observational studies encompassing 4686 patients, reporting a small survival advantage with mechanical valves. ${ }^{2}$ Before recommending a mechanical aortic valve on the basis of these data, surgeons and cardiologists should consider these 3 points:

1. Current best evidence indicates no significant difference in long-term survival after bioprosthetic versus mechanical aortic valve replacement in patients aged 50 to 70 years.

2. Transcatheter valve-in-valve has lowered the risks associated with reintervention for bioprosthetic structural valve degeneration.

3. Stroke and bleeding carry substantial mortality and morbidity and remain commoner long term after mechanical than biological valve replacement.

The single randomized trial performed in this age-group showed no significant survival difference 15 years after bioprosthetic versus mechanical aortic valve replacement, findings confirmed by 2 large observational analyses in the New England Journal of Medicine and JAMA (Figure 1). ${ }^{3-5}$ These studies successfully address the inherent confounding bias arising when specific patient

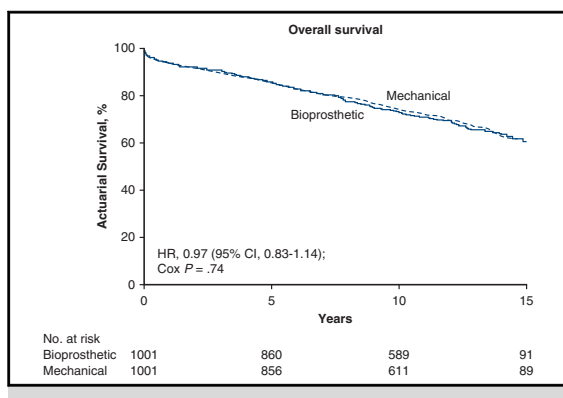

Fifteen-year survival after bioprosthetic versus mechanical aortic valve replacement.

\section{Central Message}

In patients aged 50 to 70 years, either a bioprosthetic or mechanical valve replacement remains a reasonable choice.

See Article page 706.

characteristics predict both the outcome and the choice of intervention-in this case shorter estimated life expectancy and noncompliance, both of which predict reduced survival and a bioprosthesis. Randomization is the most effective way of distributing these confounders evenly between comparison arms: consequently, a well-designed meta-analysis of randomized trials is "best evidence," and synthesizing data from multiple randomized studies is a particularly valuable approach when the available trials are underpowered and inconclusive.

However, the study by Diaz and colleagues is a meta-analysis dominated by nonrandomized studies,

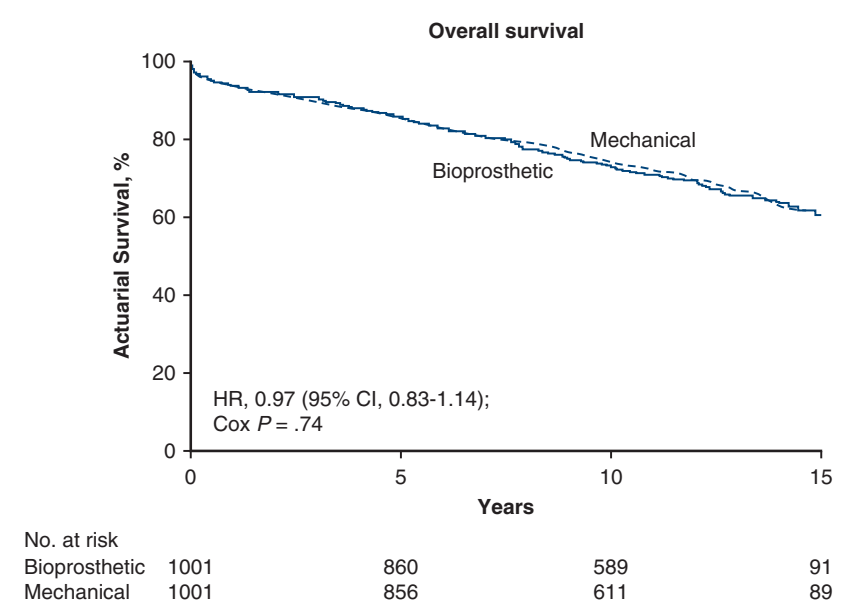

FIGURE 1. Fifteen-year survival in 2002 propensity-matched patients aged 50 to 69 years after bioprosthetic versus mechanical aortic valve replacement. ${ }^{4} H R$, Hazard ratio; $C I$, confidence interval. 
mostly limited by confounding bias favoring mechanical valves. In addition, the authors chose not to include relevant, well-designed studies such as the New England Journal of Medicine analysis. Consequently, their meta-analysis results are unreliable. Perhaps more importantly, these data no longer reflect contemporary practice, primarily because of the changing balance of risks associated with valve-related reoperation, bleeding, and embolic stroke. Transcatheter valve-in-valve has reduced mortality of reintervention for bioprosthetic degeneration for many patients, whereas the considerable mortality and morbidity associated with stroke and major bleeding (both more common after mechanical than bioprosthetic valve replacement) remain substantially unchanged. ${ }^{6}$

In summary, this meta-analysis provides no reason to deviate from clinical practice based on individual patient preference and risk factors, consensus guidelines, and best evidence. In patients aged 50 to 70 years, either a bioprosthetic or mechanical valve replacement remains a reasonable choice.

\section{References}

1. Gurevitch J, Koricheva J, Nakagawa S, Stewart G. Meta-analysis and the science of research synthesis. Nature. 2018;555:175-82.

2. Diaz R, Hernandez-Vaquero D, Alvarez-Cabo R, Avanzas P, Silva J, Moris C, et al Long-term outcomes of mechanical versus biological aortic valve prosthesis: systematic review and meta-analysis. J Thorac Cardiovasc Surg. 2019;158:706-14.e18.

3. Stassano P, Di Tommaso L, Monaco M, Lorio F, Pepino P, Spampinato N, et al Aortic valve replacement: a prospective randomized evaluation of mechanical versus biological valves in patients ages 55 to 70 years. J Am Coll Cardiol. 2009;54:1862-8.

4. Goldstone AB, Chiu P, Baiocchi M, Lingala B, Patrick WL, Fischbein MP, et al Mechanical or biologic prostheses for aortic-valve and mitral-valve replacement. N Engl J Med. 2017;377:1847-57.

5. Chiang YP, Chikwe J, Moskowitz AJ, Itagaki S, Adams DH, Egorova NN Survival and long-term outcomes following bioprosthetic vs mechanical aortic valve replacement in patients aged 50 to 69 years. JAMA. 2014 312:1323-9.

6. Tuzcu EM, Kapadia SR, Vemlapalli S, Carroll JD, Holmes DR Jr, Mack MJ, et al. Transcatheter aortic valve replacement of failed surgically implanted bioprostheses: the STS/ACC registry. J Am Coll Cardiol. 2018; $72: 370382$. 